\title{
Equation for the Nakanishi weight function using the inverse Stieltjes transform
}

\author{
V.A. Karmanov · J. Carbonell · \\ T. Frederico
}

Received: date / Accepted: date

\begin{abstract}
The bound state Bethe-Salpeter amplitude was expressed by Nakanishi in terms of a smooth weight function $g$. By using the generalized Stieltjes transform, we derive an integral equation for the Nakanishi function $g$ for a bound state case. It has the standard form $g=\hat{\mathcal{V}} g$, where $\hat{\mathcal{V}}$ is a twodimensional integral operator. The prescription for obtaining the kernel $\mathcal{V}$ starting with the kernel $K$ of the Bethe-Salpeter equation is given.
\end{abstract}

Keywords Bound states · Bethe-Salpeter equation · Nakanishi representation

\section{Introduction}

The Bethe-Salpeter (BS) approach 1 is a powerful tool to study the properties of the relativistic few-body systems. To avoid the problems related to singularities of the BS amplitude in Minkowski space, the BS equation is often transformed, by means of the Wick rotation [2, in the Euclidean space. In this way, one finds the same spectrum as for the equation in the Minkowski space. However, corresponding Euclidean BS amplitude is not enough for calculating the observables. As demonstrated in [3] in case of the ladder kernel - which displays the strongest (pole) singularities - it is possible to find the bound states and the scattering solutions in Minkowski space by a direct numerical

\footnotetext{
V.A. Karmanov

Lebedev Physical Institute, Leninsky Prospekt 53, 119991 Moscow, Russia

E-mail: karmanov@sci.lebedev.ru

J. Carbonell

Institut de Physique Nucleaire, Université Paris-Sud, IN2P3-CNRS, 91406 Orsay Cedex, France

E-mail: carbonell@ipno.in2p3.fr

T. Frederico

Instituto Tecnológico de Aeronáutica, DCTA, 12228-900, S. José dos Campos, Brazil

E-mail: tobias@ita.br
} 
calculations. However, this problem is much more complicated to deal with than in the Euclidean space.

Another efficient approach to solve the BS equation, proposed in [4] and developed in a series papers [5, 6, 7, 8, 9, 10, 11, 12, relies on the Nakanishi representation [13 of the BS amplitude $\Phi(k, p)$ in terms of a non-singular weight function $g(\gamma, z)$. This representation reads:

$$
\Phi(k, p)=\int_{0}^{\infty} d \gamma^{\prime} \int_{-1}^{1} d z^{\prime} \frac{g\left(\gamma^{\prime}, z^{\prime}\right)}{\left(\gamma^{\prime}+\kappa^{2}-k^{2}-p \cdot k z^{\prime}-i \epsilon\right)^{3}},
$$

where $\kappa^{2}=m^{2}-\frac{1}{4} M^{2}, m$ is the constituent mass and $M$ is the total mass of the bound state $\left(p^{2}=M^{2}\right)$. Once $g$ is known, one can compute the BS amplitude by means of (11) and express through $g$ the observables, like the electromagnetic form factors 14. For the massless ladder exchange $(\mu=0)$, the integral (1) turns into a one-dimensional one (over $z^{\prime}$ only). This $1 \mathrm{D}$ representation was used to solve the BS equation long ago, before inventing by Nakanishi the representation (11), in the pioneering researches by Wick [2] and Cutkosky [15.

In [5], the following double integral equation for the Nakanishi weight function $g$ was established:

$$
\int_{0}^{\infty} \frac{g\left(\gamma^{\prime}, z\right) d \gamma^{\prime}}{\left[\gamma+\gamma^{\prime}+z^{2} m^{2}+\left(1-z^{2}\right) \kappa^{2}\right]^{2}}=\int_{0}^{\infty} d \gamma^{\prime} \int_{-1}^{1} d z^{\prime} V\left(\gamma, z ; \gamma^{\prime}, z^{\prime}\right) g\left(\gamma^{\prime}, z^{\prime}\right),
$$

(written symbolically as $\hat{L} g=\hat{V} g$ ) where $V$ is expressed via the BS kernel $K$. The solution $g$ of the equation (2) was found in the case of an OBE ladder BS kernel in [5] and for the ladder plus cross-ladder one in [6. 16].

Equation (2) contains integral terms in both sides, though the integral in the l.h.-side is one-dimensional. This fact generates some instability of its numerical solution, since the discretizing of its l.h.-side results into an illconditioned matrix [5]. An equation for $g$, getting rid of the left-hand side integral term of (2), was first derived in [7]. It has the following form:

$$
g(\gamma, z)=\int_{0}^{\infty} d \gamma^{\prime} \int_{-1}^{1} d z^{\prime} \mathcal{V}\left(\gamma, z ; \gamma^{\prime}, z^{\prime}\right) g\left(\gamma^{\prime}, z^{\prime}\right) .
$$

Like in (2), the r.h.-side of the equation (3) contains the double integral. This derivation was based on the uniquenes 11 of the Nakanishi representation (1) and it was fulfilled for the ladder kernel only. The analytical expression for the kernel $\hat{\mathcal{V}}$ in eq. (3) is given in 7 and also below in Sec. 2 . The straightforward application of the derivation [7] to the same equation with a non-ladder kernel would meet some algebraic difficulties.

In a recent work 17 another method of transforming (2) in the canonical form (3) was found. It does not rely on the uniqueness of the Nakanishi

\footnotetext{
1 The uniqueness means that if the integral (1) is zero, then the function $g(\gamma, z)$ is also zero.
} 
representation nor on the ladder kernel but on using the explicit form of the inverse operator $\hat{L}^{-1}$ in equation $\hat{L} g=\hat{V} g$ for an arbitrary kernel. It was indeed noticed that the integral in l.h.-side of (2) is the generalized Stieltjes transform whose inverse operator $\hat{L}^{-1}$ was derived in [18].

We will review in this contribution the main steps of [17 and give simplified expressions for the kernel $\hat{\mathcal{V}}=\hat{L}^{-1} \hat{V}$ in (3). In addition, we will study the limit of exchanged mass $\mu \rightarrow 0$. We will show analytically that in this limit the equation (3) turns into the Wick-Cutkosky equation [2,15].

\section{Deriving the integral equation for $g$}

The results that follow are based on the observation [17, that the integral in l.h.-side of (2) can be trivially related to the Stieltjes transform which is inverted analytically [18,19. For the integral relation in the form, close to the 1.h.-side of eq. (2):

$$
f(\gamma) \equiv \int_{0}^{\infty} d \gamma^{\prime} L\left(\gamma, \gamma^{\prime}\right) g\left(\gamma^{\prime}\right)=\int_{0}^{\infty} d \gamma^{\prime} \frac{g\left(\gamma^{\prime}\right)}{\left(\gamma^{\prime}+\gamma+b\right)^{2}}
$$

denoted symbolically as $f=\hat{L} g$, a straightforward application of the inverse Stieltjes transform to (4) gives

$$
g(\gamma)=\hat{L}^{-1} f=\frac{\gamma}{2 \pi} \int_{-\pi}^{\pi} d \phi e^{i \phi} f\left(\gamma e^{i \phi}-b\right) .
$$

By applying the inverse integral transform (5) to both sides of the equation (2) , we obtain the equation for the Nakanishi weight function $g$ in the canonical form (3), where

$$
\mathcal{V}\left(\gamma, z ; \gamma^{\prime}, z^{\prime}\right)=\frac{\gamma}{2 \pi} \int_{-\pi}^{\pi} d \phi e^{i \phi} V\left(\gamma e^{i \phi}-z^{2} m^{2}-\left(1-z^{2}\right) \kappa^{2}, z ; \gamma^{\prime}, z^{\prime}\right)
$$

The relation between the original kernel $K$ appearing in the BS equation and the kernel $V$ in (2) and (6) was derived in Ref. [5]. In the case of the OBE kernel

$$
K\left(k, k^{\prime}\right)=-\frac{g^{2}}{\left(k-k^{\prime}\right)^{2}-\mu^{2}+i \epsilon}, \quad g^{2}=16 \pi m^{2} \alpha
$$

$V$ takes the form [5]:

$$
V\left(\gamma, z ; \gamma^{\prime}, z^{\prime}\right)= \begin{cases}W\left(\gamma, z ; \gamma^{\prime}, z^{\prime}\right), & \text { if }-1 \leq z^{\prime} \leq z \leq 1 \\ W\left(\gamma,-z ; \gamma^{\prime},-z^{\prime}\right), & \text { if }-1 \leq z \leq z^{\prime} \leq 1\end{cases}
$$

where:

$$
\begin{aligned}
W\left(\gamma, z ; \gamma^{\prime}, z^{\prime}\right) & =\frac{\alpha m^{2}}{2 \pi} \frac{(1-z)^{2}}{\left[\gamma+z^{2} m^{2}+\left(1-z^{2}\right) \kappa^{2}\right] b_{2}^{2}\left(b_{+}-b_{-}\right)^{3}} \\
& \times\left[\frac{\left(b_{+}-b_{-}\right)\left(2 b_{+} b_{-}-b_{+}-b_{-}\right)}{\left(1-b_{+}\right)\left(1-b_{-}\right)}+2 b_{+} b_{-} \log \frac{b_{+}\left(1-b_{-}\right)}{b_{-}\left(1-b_{+}\right)}\right]
\end{aligned}
$$




$$
\text { and } \quad \begin{aligned}
\quad b_{0} & =(1-z) \mu^{2}, \quad b_{ \pm}=-\frac{1}{2 b_{2}}\left(b_{1} \pm \sqrt{b_{1}^{2}-4 b_{0} b_{2}}\right) \\
b_{1} & =\gamma+\gamma^{\prime}-(1-z) \mu^{2}-\gamma^{\prime} z-\gamma z^{\prime}+\left(1-z^{\prime}\right)\left[z^{2} m^{2}+\left(1-z^{2}\right) \kappa^{2}\right] \\
b_{2} & =-\gamma\left(1-z^{\prime}\right)-\left(z-z^{\prime}\right)\left[(1-z)\left(1-z^{\prime}\right) \kappa^{2}+\left(z+z^{\prime}-z z^{\prime}\right) m^{2}\right]
\end{aligned}
$$

The kernel $\mathcal{V}$ of Eq. (3) is determined by inserting (86) in the integral (6).

As mentioned in the Introduction, the canonical form (3) of Eq. (2) was first derived in [7] under the hypothesis of the ladder kernel and an expression for the kernel $\mathcal{V}$ was found 2 We calculate analytically some integrals and transform the kernel [7] to the form:

$$
\mathcal{V}\left(\gamma, z ; \gamma^{\prime}, z^{\prime}\right)=+\frac{\alpha m^{2}}{2 \pi} \times \begin{cases}h\left(\gamma,-z ; \gamma^{\prime},-z^{\prime}\right), & \text { if }-1 \leq z^{\prime} \leq z \leq 1 \\ h\left(\gamma, z ; \gamma^{\prime}, z^{\prime}\right), & \text { if }-1 \leq z \leq z^{\prime} \leq 1\end{cases}
$$

The function $h$ is obtained from eqs. (27), (28) in [7]:

$$
h\left(\gamma, z ; \gamma^{\prime}, z^{\prime}\right)=Q\left(\gamma^{\prime}, z^{\prime}\right)+\theta(\eta) P\left(\gamma, z ; \gamma^{\prime}, z^{\prime}\right) .
$$

The first term $Q$ is given by

$$
Q\left(\gamma^{\prime}, z^{\prime}\right)=\int_{0}^{\infty} \chi(y) d y=-\frac{A^{\prime}}{A A_{s}}-\left\{\begin{array}{l}
\frac{2 \mu^{2}}{A_{s}^{3 / 2}}\left(2 \arctan \frac{A^{\prime}}{\sqrt{A_{s}}}-\pi\right), \text { if } A_{s}>0 \\
\frac{2 \mu^{2}}{\left|A_{s}\right|^{3 / 2}} \log \frac{A^{\prime}+\sqrt{\left|A_{s}\right|}}{A^{\prime}-\sqrt{\left|A_{s}\right|}}, \text { if } A_{s}<0
\end{array}\right.
$$

where the integrand is given by 3

$$
\chi(y)=\frac{y^{2}}{\left(A y^{2}+A^{\prime} y+\mu^{2}\right)^{2}}
$$

and $A_{s}=4 A \mu^{2}-A^{\prime 2}, A=\gamma^{\prime}+m^{2}-\frac{1}{4}\left(1-z^{\prime 2}\right) M^{2}, A^{\prime}=\gamma^{\prime}+\mu^{2}$

The argument $\eta$ of the theta-function in the second term in (11) reads

$$
\eta=\gamma \frac{1+z^{\prime}}{1+z}-\mu^{2}-\gamma^{\prime}-2 \mu \sqrt{\gamma^{\prime}+m^{2}-\frac{1}{4}\left(1-z^{\prime 2}\right) M^{2}}=-B-2 \mu \sqrt{A} .
$$

The function $P\left(\gamma, z ; \gamma^{\prime}, z^{\prime}\right)$ in (11) has the form:

$$
P\left(\gamma, z ; \gamma^{\prime}, z^{\prime}\right)=\frac{B}{\gamma A \Delta} \frac{1+z}{1+z^{\prime}}-C
$$

where

$$
B=-\gamma \frac{1+z^{\prime}}{1+z}+\mu^{2}+\gamma^{\prime}, \quad \Delta=\sqrt{B^{2}-4 \mu^{2} A}
$$

${ }^{2}$ Following 20, a misprint in the kernel sign in 7 ] was corrected here and in [17.

3 A misprint in eq. (28) of [17] for $\chi(y)$ was corrected in [12):

$$
\chi(y)=\frac{y^{2}}{\left(A+y^{2}+A^{\prime} y+\mu^{2}\right)^{2}} \rightarrow \chi(y)=\frac{y^{2}}{\left(A y^{2}+A^{\prime} y+\mu^{2}\right)^{2}} .
$$




$$
\begin{gathered}
C=\int_{y_{-}}^{y_{+}} \chi(y) d y=\hat{\chi}\left(y_{+}\right)-\hat{\chi}\left(y_{-}\right), \quad y_{ \pm}=\frac{-B \pm \Delta}{2 A} \\
\hat{\chi}(y)=\frac{A^{\prime} \mu^{2}-2 A \mu^{2} y+A^{\prime 2} y}{A A_{s}\left[\mu^{2}+y\left(A^{\prime}+A y\right)\right]}+ \begin{cases}\frac{4 \mu^{2}}{A_{s}^{3 / 2}} \arctan \frac{A^{\prime}+2 A y}{\sqrt{A_{s}}}, & \text { if } A_{s}>0 \\
\frac{2 \mu^{2}}{\left|A_{s}\right|^{3 / 2}} \log \frac{\frac{A^{\prime}+2 A y}{\sqrt{\left|A_{s}\right|}}+1}{\frac{A^{\prime}+2 A y}{\sqrt{\left|A_{s}\right|}}-1}, & \text { if } A_{s}<0\end{cases}
\end{gathered}
$$

Kernel $\mathcal{V}$ is determined above by two quite different expressions: eq. (6) found in 17 and eq. (10) found in 77. It has not been possible to prove their identity analytically but only by their numerical comparison [17. It is worth noticing however that, contrary to $V$, kernel $\mathcal{V}$ displays some singularities in variable $\gamma$ and requires some careful treatment.

The coupling constants $\alpha$ and the Nakanishi functions $g(\gamma, z)$ for selected values of $M$ and $\mu$ found in 7 from equations (2) and (3) coincide with each other within numerical accuracy as well as with the Euclidean BS results.

\section{The limit $\mu \rightarrow 0$}

In the $\mu=0$ case, which constitutes the original Wick-Cutkosky model 2, 15, kernel (8) obtains a simple analytical form:

$$
\begin{aligned}
V\left(\gamma, z ; \gamma^{\prime}, z^{\prime}\right)= & \frac{\alpha m^{2}}{2 \pi} \frac{1}{\left[\gamma+z^{2} m^{2}+\left(1-z^{2}\right) \kappa^{2}\right]\left[\gamma^{\prime}+z^{\prime 2} m^{2}+\left(1-z^{\prime 2}\right) \kappa^{2}\right]} \\
& \times \begin{cases}\frac{1}{\left[\gamma+\gamma^{\prime} \frac{(1+z)}{\left(1+z^{\prime}\right)}+z^{2} m^{2}+\left(1-z^{2}\right) \kappa^{2}\right]} \frac{(1+z)}{\left(1+z^{\prime}\right)}, & \text { if } z<z^{\prime} \\
\frac{1}{\left[\gamma+\gamma^{\prime} \frac{(1-z)}{\left(1-z^{\prime}\right)}+z^{2} m^{2}+\left(1-z^{2}\right) \kappa^{2}\right]} \frac{(1-z)}{\left(1-z^{\prime}\right)}, & \text { if } z>z^{\prime}\end{cases}
\end{aligned}
$$

Inserting this expression in (6) and integrating over $\phi$ one gets for $\mathcal{V}$ :

$\mathcal{V}\left(\gamma, z ; \gamma^{\prime}, z^{\prime}\right)=\frac{\alpha m^{2}}{2 \pi \gamma^{\prime}} \frac{1}{\left[\gamma^{\prime}+m^{2}-\frac{1}{4}\left(1-{z^{\prime}}^{2}\right) M^{2}\right]} \times \begin{cases}\theta\left(\gamma^{\prime} \frac{1+z}{1+z^{\prime}}-\gamma\right), & \text { if } z<z^{\prime} \\ \theta\left(\gamma^{\prime} \frac{1-z}{1-z^{\prime}}-\gamma\right), & \text { if } z>z^{\prime}\end{cases}$

Following [5], we look for a solution of (3) in the form

$$
\bar{g}(\gamma, z)=\delta(\gamma) g(z) .
$$

This form is justified provided the equation for $g(z)$ does not depend on $\gamma$. Substituting the former expression in (3), we obtain:

$$
\delta(\gamma) g(z)=\int_{0}^{\infty} d \gamma^{\prime} \int_{-1}^{1} d z^{\prime} \mathcal{V}\left(\gamma, z ; \gamma^{\prime}, z^{\prime}\right) \delta\left(\gamma^{\prime}\right) g\left(z^{\prime}\right)
$$


Integrating both sides of (17) over $\gamma$ we find:

$$
g(z)=\int_{-1}^{1} d z^{\prime} \widetilde{V}\left(z, z^{\prime}\right) g\left(z^{\prime}\right)
$$

with

$$
\widetilde{V}\left(z, z^{\prime}\right)=\frac{\alpha m^{2}}{2 \pi} \frac{1}{\left[m^{2}-\frac{1}{4}\left(1-z^{\prime 2}\right) M^{2}\right]} \begin{cases}\frac{1+z}{1+z^{\prime}}, & \text { if } z<z^{\prime} \\ \frac{1-z}{1-z^{\prime}}, & \text { if } z<z^{\prime}\end{cases}
$$

which exactly coincides with the Wick-Cutkosky equation 2, 15. Notice that the same limit $\mu \rightarrow 0$ can be derived starting from (3) and kernel (10).

\section{Conclusion}

Starting with an equation for the Nakanishi weight function $g$ of the BetheSalpeter amplitude in the form $\hat{L} g=\hat{V} g$, previously established in [5], and using an analytic inversion of the Stieltjes integral transform, we transformed this equation in the canonical form $g=\hat{\mathcal{V}} g$.

This result generalizes, to an arbitrary kernel given by a set of irreducible Feynman graphs, the equation found in [7] which was stablished for a ladder kernel. This generalization strongly expands the applicability of the Nakanishi representation to find the solution of the Bethe-Salpeter equation, as well as its applications. Equation (3) can be extended straightforwardly to the twofermion system starting, for example, from 9 .

The possibility to apply an analytic inversion of the Stieltjes transform can be useful in other fields of nuclear and hadronic physics, where the use of integral transforms was pioneered by [21,22,23], in order to avoid the instabilities of the numerical inversion.

\section{Aknowledgements}

We are indebted to G. Salmé for useful discussions. T.F. thanks CNPq, CAPES and FAPESP of Brazil. V.A.K. thanks the support of FAPESP, the grant \#2015/22701-6 and is sincerely grateful for kind hospitality to Theoretical Nuclear Physics Group in ITA, São José dos Campos, Brazil, where the main part of this research was carried out.

\footnotetext{
${ }^{4}$ On the one hand, the integrand in (17) contains the expression $\frac{1}{\gamma^{\prime}} \delta\left(\gamma^{\prime}\right)$ which is infinite. On the other hand, the integration of r.h.-side of (17) over $\gamma$ gives, due to the theta-function, the factor $\gamma^{\prime} \frac{1 \pm z}{1 \pm z^{\prime}} \cdot \gamma^{\prime}$ from this factor cancels $\gamma^{\prime}$ in the denominator. In this way, we obtain a finite and certain result for the product $\infty \cdot 0$.
} 


\section{References}

1. E.E. Salpeter, H.A. Bethe, A Relativistic Equation for Bound-State Problems, Phys. Rev. 84, 1232 (1951).

2. G.C. Wick, Properties of the Bethe-Salpeter Wave Functions, Phys. Rev. 96, 1124 (1954).

3. J. Carbonell, V.A. Karmanov, Solving Bethe-Salpeter scattering state equation in Minkowski space, Phys. Rev. D 90, 056002 (2014).

4. K. Kusaka and A.G. Williams, Solving the Bethe-Salpeter equation for scalar theories in Minkowski space, Phys. Rev. D 51 (1995) 7026;

K. Kusaka, K. Simpson and A.G. Williams, Solving the Bethe-Salpeter equation for bound states of scalar theories in Minkowski space, Phys. Rev. D 56, 5071 (1997).

5. V.A. Karmanov and J. Carbonell, Solving Bethe-Salpeter equation in Minkowski space, Eur. Phys. J. A 27, 1 (2006).

6. J. Carbonell, V.A. Karmanov, Cross-ladder effects in Bethe-Salpeter and Light-Front equations, Eur. Phys. J. A 27, 11 (2006).

7. T. Frederico, G. Salmè and M. Viviani, Quantitative studies of the homogeneous BetheSalpeter equation in Minkowski space, Phys. Rev. D 89, 016010 (2014).

8. T. Frederico, G. Salmè and M. Viviani, Two-body scattering states in Minkowski space and the Nakanishi integral representation onto the null plane, Phys. Rev. D 85, 036009 (2012); Solving the inhomogeneous Bethe-Salpeter Equation in Minkowski space: the zero-energy limit, Eur. Phys. J. C 75, 398 (2015).

9. W. de Paula, T. Frederico, G. Salmè, M. Viviani, Advances in solving the two-fermion homogeneous Bethe-Salpeter equation in Minkowski space, Phys.Rev. D 94, 071901 (2016).

10. C. Gutierrez, V. Gigante, T. Frederico, G. Salmè, M. Viviani, L. Tomio, Bethe-Salpeter bound-state structure in Minkowski space, Phys. Lett. B 759, 131 (2016).

11. T. Frederico, J. Carbonell, V. Gigante and V.A. Karmanov, Inverting the Nakanishi integral relation for a bound state Euclidean Bethe-Salpeter amplitude, Few-Body Syst. 56, 549 (2016).

12. T. Frederico, J. Carbonell, and V.A. Karmanov, Euclidean to Minkowski Bethe-Salpeter amplitude and observables, Eur. Phys. J. C 77, 58 (2017).

13. N. Nakanishi, Partial-Wave Bethe-Salpeter Equation, Phys. Rev. 130 (1963) 1230; General Survey of the Theory of the Bethe-Salpeter Equation, Prog. Theor. Phys. Suppl. 43, 1 (1969); Graph Theory and Feynman Integrals (Gordon and Breach, New York, 1971).

14. J. Carbonell, V.A. Karmanov and M. Mangin-Brinet, Electromagnetic form factor via Bethe-Salpeter amplitude in Minkowski space, Eur. Phys. J. A 39, 53 (2009).

15. R.E. Cutkosky, Solutions of the Bethe-Salpeter Equation, Phys. Rev. 96, 1135 (1954).

16. V. Gigante, J.H. Alvarenga Nogueira, E. Ydrefors, C. Gutierrez, V.A. Karmanov, and T. Frederico, Bound state structure and electromagnetic form factor beyond the ladder approximation, Phys. Rev. D 95, 056012 (2017).

17. J. Carbonell, T. Frederico, V.A. Karmanov, Bound state equation for the Nakanishi weight function, Phys. Lett. B, 769, 418 (2017).

18. J.H. Schwarz, The generalized Stieltjes transform and its inverse, Jour. of Math. Phys. 46, 014501 (2005); arXiv:math-ph/0405050v1.

19. D.B. Sumner, An inversion formula for the generalized Stieltjes transform, Bull. Am. Math. Soc. 55, 174 (1949).

20. G. Salmè, private communication, November, 2016.

21. V.D. Efros, Calculation of inclusive transition spectra and the reaction cross sections without wave functions, Sov. J. Nucl. Phys. 41, 949 (1985).

22. V. D. Efros, W. Leidemann, G. Orlandini, and N. Barnea, The Lorentz integral transform (LIT) method and its applications to perturbation-induced reactions, J. Phys. G: Nucl. Part. Phys. 34, R459 (2007)

23. G. Orlandini, F. Turro, Integral transform methods: a critical review of various kernels, Few Body Syst. 58, 76 (2017). 